\title{
The Importance of Culinary-Medicinal Mushrooms from Ancient Times to the Present
}

\author{
Solomon P. Wasser \\ Institute of Evolution, University of Haifa, Mt. Carmel, Haifa 31905, Israel; N.G. Kholodny \\ Institute of Botany, NASU, Tereshchenskivska 2, Kiev, 01001, Ukraine
}

Higher Basidiomycetes mushrooms have been used in folk medicine throughout the world since ancient times. For millennia, mushrooms have been valued by humankind as edible and medicinal resources. Traditional use of mushrooms as medicines has been long established among different ethnic groups. In the Far East countries, especially in China, Japan, and Korea, mushrooms have long been revered for their curative attributes.

Paramount among these is Ganoderma lucidum (W. Curt.: Fr.) Lloyd (Ling zhi in Chinese; Reishi, Mannentake, or Sachitake in Japanise; and Youngzhi in Korea). It is valued for both its medicinal and spiritual properties. For centures, this mushroom has been regarded in the Orient as an effective medicinal source. It is also considered a symbol of happy augury and good fortune, good health, longevity, and even life with the immortals. Very important Far East culinary-medicinal mushrooms are also Lentinus edodes (Berk.) Singer (Shiitake mushroom) and Trametes versicolor (L.: Fr.) Pilát (Turkey Tail).

Mushrooms have played an important role as cures for ailments affecting the rural populations of Russia and other European Slavic countries. The most important species are Inonotus obliquus (Pers.: Fr.) Pilát (Chaga), Fomitopsis officinalis (Vill.: Fr.) Bond. et Singer, and Fomes fomentarius Fr.: Fr. for treating gastrointestinal disorders, various forms of cancers, bronchial asthma, night sweats, etc. There is also a long record of traditional use of mushrooms as curatives in Mesoamarica (especially species of the genus Psilocybe).

The number of mushrooms on Earth is estimated at 140,000 , yet perhaps only 10\% (approximately 14,000 named species) are known. Edible higher Basidiomycetes are being evaluated for their nutritional value and acceptability, as well as their pharmacological properties. They make up a vast and yet largely untapped source of powerful new pharmaceutical products. In particular, and most importantly for modern medicine, they present an unlimited source of polysaccharides with anticancer and immunostimulating properties. Many, if not all, Basidiomycetes mushrooms contain biologically active polysaccharides in their fruit bodies, cultured mycelia, and culture broth.

The data about mushroom polysaccharides are summarized for 651 species and seven intraspecific taxa from 182 genera of higher Hetero- and Homobasidiomycetes. These polysaccharides are of different chemical composition; the main ones comprise the group of $\beta$-glucans. $\beta-(1 \rightarrow 3)$ linkages in the main chain of the glucan and further $\beta-(1 \rightarrow$ 6) branch points are needed for their antitumor action. Numerous bioactive polysaccharides or polysaccharide-protein complexes from medicinal mushrooms are described that appear to enhance innate and cell-mediated immune responses and exhibit antitumor activities in animals and humans.

Stimulation of host immune defense systems by bioactive polymers from medicinal mush- 
rooms has significant effects on the maturation, differentiation, and proliferation of many kinds of immune cells in the host. Many of these mushroom polymers were reported previously to have immunotherapeutic properties by facilitating growth inhibition and destruction of tumor cells. While the mechanism of their antitumor actions is still not completely understood, stimulation and modulation of key host immune responses by these mushroom polymers appears central. Recent evidence suggests that mushroom polymers ( $\beta$-glucans) may trigger the stimulation of many kinds of immune cells in animals and humans. Several of the mushroom polysaccharide compounds (lentinan, krestin (PSK), PSP, schizophyllan, befungin) have proceeded through Phases I, II, and III clinical trials and are used extensively and successfully to treat various cancers and other diseases in Asia, but not in many Western countries, because in many cases the standards of these trials may not meet current Western regulatory requirements. The polysaccharides of some other promising medicinal mushrooms species-Agaricus brasiliensis $\mathrm{S}$. Wasser et al., Phellinus linteus (Berk. et W. Curt.) Teng, Grifola frondosa (Dicks.: Fr.) S.F. Gray, Tremella mesenterica Retz.: Fr., Hypsizygus marmoreus (Peck) Bigel., and Flammulina velutipes (W. Curt.: Fr.)P. Karst.—also show good results.

In the second half of the $20^{\text {th }}$ century, mushroom-producing technologies grew enormously. Mushrooms represent a valuable source of bioactive agents with potent and unique medicinal properties.
Some of recently isolated and identified substances of Higher Basidiomycetes mushroom origin express promising antitumor, immune modulating, antioxidant, cardiovascular, antihypercholesterolemia, antiviral, antibacterial, antiparasitic, hepatoprotective, and antidiabetic effects.

Many of them are not strictly pharmaceutical products (real medicines) but represent a novel class of dietary supplements (DSs) or "mushroom nutriceuticals." The agents derived from medicinal mushroom fruit bodies, cultured mycelium, and/or culture filtrates exert a wide range of beneficial biological effects when tested in vitro or using animal models. Recent years have seen a surge of commercial interest in medicinal mushroom DSs, the common market value of which is approximately $\$ 13$ billion US dollars.

The safety advantages of using mushroom-based DSs, as opposed to herbal preparations are the following. (1) The overwhelming majority of mushrooms used for production of DSs are cultivated commercially (and not gathered in the wild); this provides a very good chance of proper identification, and pure and unadulterated products. In many cases it also means genetic uniformity. (2) Mushrooms are easily propagated vegetatively and thus keep to one clone. The mycelium can be stored for a long time, and the genetic and biochemical consistency may be checked after considerable time. (3) The main advantage, in our opinion, is that many mushrooms are capable of growing in the form of mycelia biomass in submerged cultures. 